\title{
Deep blue organic light-emitting diodes of 1,8-diaryl anthracene
}

\author{
PRABHJYOT BHUI ${ }^{\mathrm{a}}$, QAMAR TABREZ SIDDIQUI ${ }^{\mathrm{b}, \mathrm{c}}$, MOHAMMAD MUNEER $^{\mathrm{c}}$, \\ NEERAJ AGARWAL ${ }^{\mathrm{b}, *}$ and SANGITA BOSE ${ }^{\mathrm{a}, *}$ \\ ${ }^{a}$ School of Physical Sciences, UM-DAE Centre for Excellence in Basic Sciences, University of Mumbai, Kalina, \\ Santacruz (E), Mumbai, Maharashtra 400 098, India \\ ${ }^{\mathrm{b}}$ School of Chemical Sciences, UM-DAE Centre for Excellence in Basic Sciences, University of Mumbai, \\ Kalina, Santacruz (E), Mumbai, Maharashtra 400 098, India \\ ${ }^{\mathrm{c}}$ Department of Chemistry, Aligarh Muslim University, Aligarh 202 002, India \\ E-mail: na@cbs.ac.in; sangita@cbs.ac.in
}

MS received 6 July 2018; revised 28 September 2018; accepted 5 October 2018; published online 27 November 2018

\begin{abstract}
We report on the optimization of organic light emitting diode (OLED) devices using 1,8-di-(4trifluromethylphenyl)-anthracene (CF3-DPA) as the active emissive layer. CF3-DPA emits in the deep blue region with an emission peak at $432 \mathrm{~nm}$ in solution which showed a slight red shift in thin films. CF3-DPA has high reported fluorescence quantum efficiency, $\sim 67 \%$, as compared to 9,10-diphenyl anthracene (9,10-DPA). We optimized the OLED devices with different hole transporting layers (HTLs). Bilayer devices formed with $N, N^{\prime}$-di(1-naphthyl)- $N, N^{\prime}$-diphenyl-(1, $1^{\prime}$-biphenyl)-4, $4^{\prime}$-diamine (NPD) as the HTL gave a reasonable light output. We observed that trilayer or multilayer devices with the inclusion of poly(3,4-ethylene dioxythiophene)poly(styrene sulfonate) (PEDOT:PSS) and/or copper phthalocyanine as an additional HTL reduced the turn on voltage by $\sim 5$ to $9 \mathrm{~V}$, though the brightness of the light emission also decreased. Including suitable carrier (electron or hole) transporting layers like 2, $2^{\prime}, 2^{\prime \prime}$-(1,3,5-Benzinetriyl)-tris(1-phenyl-1-H-benzimidazole) (TPBi) and 4,4'-Bis( $N$-carbazolyl)-1, $1^{\prime}$-biphenyl (CBP) increases the efficiency of the devices. From our studies, we conclude that though NPD/CF3-DPA interface is crucial for light emission, the performance of the devices is limited by the mismatch of the hole and electron mobilities and the low internal quantum efficiency of CF3-DPA in the solid state. Devices having ITO/NPD/CF3-DPA/TPBi/LiF-Al geometry were observed to be the most efficient.
\end{abstract}

Keywords. Anthracene derivative; blue organic light emitting device; hole transporting layer.

\section{Introduction}

Organic light-emitting diodes (OLEDs) have been a focus of recent research and development due to their potential for use in the new type of large-area, flexible flat-panel displays for mobiles and televisions. ${ }^{1-4}$ However, one of the major concerns in the fabrication of full-colour OLED displays is the equal performance of the three primary colors of red, green and blue. It has been successfully demonstrated that efficient red or green OLEDs can be made by using vacuum-deposition of small molecules of organic electroluminescent materials or spin-coating of conjugated polymers. ${ }^{4}$ However, since blue organic materials have a larger band gap, they have a lower affinity for the electron from the cathode in

\footnotetext{
*For correspondence
}

OLEDs. Therefore, the performance of blue emitters is usually inferior to that of green or red emitters. Though the pure blue material was commercialized for the first active display in 2004, the external quantum efficiency (EQE) of the blue devices has been showing a steady increase. ${ }^{5,6}$ In spite of being able to obtain a reasonably high EQE, long-term stability and color purity of the blue OLEDs require improvement and efforts are on to find newer blue emitting materials with desired properties. $^{7}$

Anthracene in solution exhibits fluorescence in the blue spectral region with high quantum efficiency making it and its derivatives good candidates for efficient blue emitters. ${ }^{8}$ However, they are prone to aggregation in thin films, owing to their highly planar structure. ${ }^{9,10}$ Aggregation of anthracene in thin films lead to the quenching of the fluorescence and redshift in 
its emission maxima. Among anthracene derivatives, 9,10-diphenylanthracene (DPA) might be thought to be a promising material as a blue emitter since DPA has a photoluminescence (PL) quantum yield close to unity. However, a weak blue electroluminescence (EL) emission, about $0.09 \mathrm{~cd} / \mathrm{m}^{2}$ at $100 \mathrm{~mA} / \mathrm{cm}^{2}$, from bilayer devices consisting of a DPA emitting layer and a diamine hole transporting layer (HTL) layer have been reported. ${ }^{8}$ Thereafter, it has been shown that using multi-layer geometry for the devices with different combinations of HTL and electron transporting layer (ETL) or doping DPA with bathocuproine (BCP) improves the luminous efficiency marginally. About a couple of years back, a new series of undoped 9,10-DPA-based blue fluorophores was synthesized with bulky substituents which showed much higher EQE of $4.5 \%{ }^{11}$ as compared to unsubstituted DPA (EQE-1.5\%) or doped 9,10-DPA $(\mathrm{EQE} \sim 2 \%) .{ }^{12}$ Subsequently, several attempts were made to improve the emission and thin film properties of anthracene derivatives. ${ }^{13-18}$ It was demonstrated that the film forming properties of DPA can be improved by introducing bulky aryl substituents at the 2- and 6-positions of anthracene (instead of the 9- and 10positions). ${ }^{19}$ Recently, the synthesis of derivatives of the 1,8-diaryl anthracene have been reported. ${ }^{20,21}$ These anthracene derivatives were reported to emit in the blue region and showed high fluorescence quantum efficiency. One of the derivatives also exhibited surfactantassisted nano-self-assembly forming nano-ribbons and nano-plates. It was proposed that these compounds with their blue emitting properties and compatible HOMO-LUMO energy levels would be suitable to form OLEDs.

In this paper, we report on the morphological and electro-optical properties of thin films formed of an anthracene derivative, 1,8-bis-(4-trifluromethylphenyl)anthracene (CF3-DPA), synthesized according to literature. ${ }^{20}$ Fluorescence peak at $432 \mathrm{~nm}$, high quantum efficiency ( $\sim 67 \%$ with respect to 9,10 -DPA) and good thin film forming properties of CF3-DPA allow us to study it in OLED applications. We fabricated a series of devices with different structures having different HTLs and studied the effect of them on the turn on voltage $\left(\mathrm{V}_{\mathrm{ON}}\right)$ and luminance of the devices. We observed that $N, N^{\prime}$-Di(1-naphthyl)- $N, N^{\prime}$-diphenyl-(1,1'-biphenyl)-4,4'-diamine or NPD was the most suited HTL with respect to light output. The device of ITO/NPD/CF3DPA/LiF-Al gave blue light with CIE chromaticity of $(0.14,0.04)$. However, the turn on voltage $\left(\mathrm{V}_{\mathrm{ON}}\right)$ was quite high, about $13 \mathrm{~V}$. We observed that multilayer devices having a layer of poly(3,4-ethylene dioxythiophene) poly(styrene sulfonate) (PEDOT:PSS) before NPD reduces the $\mathrm{V}_{\mathrm{ON}}$ considerably, though the light output is reduced. The efficiency of the devices could be enhanced by using a suitable carrier (electron and/or hole) transporting layers like TPBi and CBP. Devices having ITO/NPD/CF3-DPA/TPBi/LiFAl geometry were observed to be the most efficient at a current density of $50 \mathrm{~mA} / \mathrm{cm}^{2}$ while devices having ITO/PEDOT:PSS/CF3-DPA/TPBi/LiF-Al geometry were most efficient at a low current density of $1 \mathrm{~mA} / \mathrm{cm}^{2}$, though they were not very stable. Thus, we show that 1,8-diaryl anthracene derivatives are good candidates for deep blue OLEDs.

\section{Experimental}

Trifluoromethyl substituted 1,8-diaryl anthracene was synthesized using the Suzuki-Miyaura coupling reaction of 1,8-dicholoroanthracene using the catalyst Pd-PEPPSI-iPr. The details of the synthesis are reported earlier. ${ }^{21}$ The structure of the synthesized compound is shown in Figure 1(a). The photo-physical characterization of the synthesized compound was carried out in solution as well as in solid thin films using SHIMADZU UV180 spectrophotometer and HORIBA FLUOROLOG 3 fluorimeter. The morphology of the thin films was studied by atomic force microscope (AFM).

All OLED devices were grown on ITO coated glass with a surface resistivity of $15-25 \Omega / \mathrm{sq}$ (commercially brought from Sigma Aldrich) which were patterned to make four devices on a substrate of $2 \times 1 \mathrm{~cm}^{2}$. The ITO substrates were cleaned properly with soap solution, distilled water, propanol and trichloroethylene. Finally, ozone surface treatment was given by keeping the substrates under UV light for $30 \mathrm{~min}$ which is known to reduce the work function of ITO. ${ }^{22}$ Vacuum deposition was used in the preparation of all the OLED devices. Apart from PEDOT:PSS all the other materials were thermally evaporated in a base vacuum of $5 \times 10^{-6}$ mbar. PEDOT:PSS was spin-coated at $8000 \mathrm{rpm}$ for $40 \mathrm{~s}$ and heated at $160^{\circ} \mathrm{C}$ for 30 min to give a $\sim 40 \mathrm{~nm}$ thick layer. ${ }^{23}$ The thickness of each layer deposited by thermal evaporation was measured using an in situ quartz crystal monitor. In addition, the thickness of each material was independently calibrated ex situ using a surface profilometer. The final electrode or cathode of the devices formed by $\mathrm{LiF} / \mathrm{Al}$ was deposited using a shadow mask that defined the active area of the OLED devices which were in the range of $6-25 \mathrm{~mm}^{2}$. The thickness of $\mathrm{LiF}$ was $\sim 1 \mathrm{~nm}$ while that of $\mathrm{Al}$ was $\sim 160 \mathrm{~nm}$ for all the devices. The electro-luminance spectroscopy of the devices were measured using a set up consisting of a Bausch and Lomb 350-750 nm monochromator and Hamamatsu R212 photomultiplier tube (PMT) as the detector. The current-voltage (I-V) characteristics were measured using the 2400 Keithley source meter at $\lambda=439 \mathrm{~nm}$ and the luminance-voltage $(\mathrm{L}-\mathrm{V})$ spectra were simultaneously recorded using lock-in detection. The lockin signal was converted to light units using the conversion factors which were estimated using a calibrated red LED. 


\section{Results and Discussion}

\subsection{Characterization of CF3-DPA}

Photophysical studies of CF3-DPA were carried out in solution and thin films using absorbance and fluorescence spectroscopy. Absorption maxima was obtained at $394 \mathrm{~nm}$ and the emission maxima at $432 \mathrm{~nm}$ in toluene. The quantum yield in solution was about $67 \%$ taking 9,10-DPA as the reference. ${ }^{20}$ The emission was measured in both spin-coated and evaporated thin films. The emission peak showed a bathochromic shift of $7 \mathrm{~nm}$ as compared to the solution (Figure 1(b)). This marginal bathochromic shift indicates little aggregation in thin films of CF3-DPA. ${ }^{24}$

Morphology of thin films of the active emissive layer plays a crucial role for OLED devices. Surface morphology of CF3-DPA thin films, which were both spin coated and grown by thermal evaporation, was studied by AFM. The AFM images of these films are shown in Figure 1(c) and (d). The surface roughness of the evaporated films was less than that of the spin-coated ones and was of the order of $\sim 2 \mathrm{~nm}$. Thus, thermally evaporated films would be more suited to form OLED devices as smother interfaces are expected. The HOMO and LUMO energy levels were estimated using the

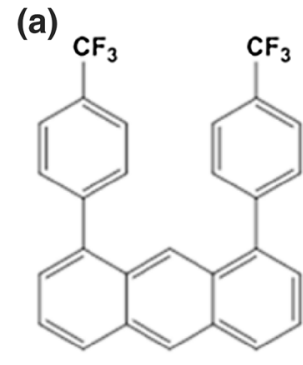

CF3-DPA

(c)

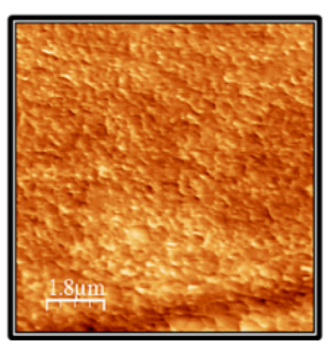

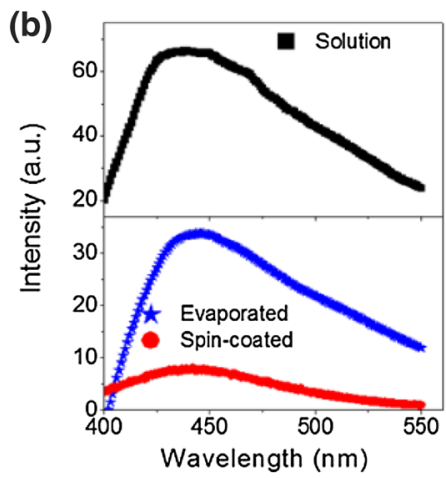

(d)

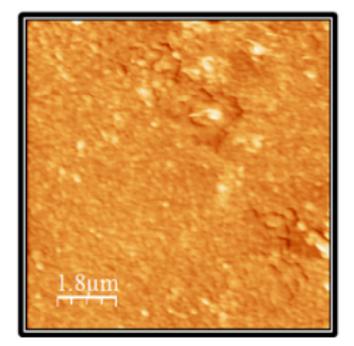

Figure 1. (a) The structure of trifluoromethyl substituted 1,8-diaryl anthracene (CF3-DPA); (b) Emission spectra of CF3-DPA in solution (black square), evaporated thin film (blue star) and spin coated thin film (red circle); (c) and (d) AFM images $(4.8 \mu \mathrm{m} \times 4.8 \mu \mathrm{m})$ of the spin-coated and evaporated thin films, respectively. cyclic voltammetry and were found to be -5.7 and $-2.8 \mathrm{eV}$, respectively. The band gap for CF3-DPA was obtained as $2.9 \mathrm{eV}$ which corresponds to $428 \mathrm{~nm}$. The $\mathrm{E}_{\mathrm{LUMO}}$ is very close to the widely used organic green emitter, tris-(8-hydroxyquinolinato)-aluminium $\left(\mathrm{Alq}_{3}\right)$ whose OLEDs have been optimized to give a high external quantum efficiency of $\sim 12 \%$ at $100 \mathrm{Cd} / \mathrm{m}^{2}$. ${ }^{12}$

\subsection{Characterization of CF3-DPA OLED devices}

PEDOT: PSS is a widely used HTL in OLEDs. Since this has $\mathrm{E}_{\text {Hомо }}$ closer to that of the plasma treated ITO, hole injection efficiency is expected to be high ${ }^{25}$ which might result in a low turn on voltage $\left(\mathrm{V}_{\mathrm{ON}}\right)$. Bilayer devices, ITO/PEDOT:PSS/CF3-DPA/LiF-Al, whose energy band diagram is shown in Figure 2(a) were fabricated. The thickness of PEDOT:PSS and CF3DPA layers were $\sim 50 \mathrm{~nm}$ each. Figure $2(\mathrm{c})$ shows the plot between current density $(\mathrm{J})$ and bias voltage $(\mathrm{V})$.

A non-zero current density $(\mathrm{J})$ was obtained at $\sim 2.5 \mathrm{~V}$ and a distinct change of slope at $\sim 5.0 \mathrm{~V}$ while no light output was detected at this bias voltage (Figure 2(d)) which could be due to the non-recombination of the carriers. To improve hole transport, we studied the effect of adding NPD, another HTL, in the device. Interestingly, for these trilayer devices, ITO/PEDOT:PSS/NPD/CF3DPA/LiF-Al (energy band diagram is shown in Figure 2(b)), the $\mathrm{J}-\mathrm{V}$ curve showed a non-zero value of $\mathrm{J}$ at a voltage of $2.5 \mathrm{~V}$ (Figure 2(c)). This was similar to the bilayer device. However, a further rise of $\mathrm{J}$ was observed at a voltage of $7.5 \mathrm{~V}$ at which light was also detected implying that $\mathrm{V}_{\mathrm{ON}}=7.5 \mathrm{~V}$ for this device. At $\mathrm{J}=50 \mathrm{~mA} / \mathrm{cm}^{2}$, the light output was quite low $\sim 2 \mathrm{Cd} / \mathrm{m}^{2}$ as shown in Figure 2(d).

We tried to find the most optimal choice of HTL so that the $\mathrm{V}_{\mathrm{ON}}$ is minimized and the light output is maximized for the devices. As the light output was quite low in PEDOT:PSS/NPD based devices, we studied the effect of another well-known HTL, copper phthalocyanine $(\mathrm{CuPc})$ on the device performance which is also known to enhance hole injection efficiency from ITO. ${ }^{26}$ Since, the NPD/CF3-DPA interface was important for light output, we made trilayer and multilayer devices retaining this interface. Devices with the following geometries ITO/CuPc/NPD/CF3-DPA/LiF$\mathrm{Al}$ and ITO/CuPc/PEDOT:PSS/NPD/CF3-DPA/LiF-Al (energy band diagrams given in Figure 3(a) and (b), respectively) were made where the thickness of $\mathrm{CuPc}$ was kept at $\sim 40 \mathrm{~nm}$. The thicknesses of PEDOT:PSS and CF3-DPA were kept at $\sim 50 \mathrm{~nm}$ as the previous devices. Figure 3(c) shows the J-V for both these devices and Figure 3(d) shows the L-V for the same two devices. Multilayer devices (Figure 3(b)) with PEDOT:PSS had 

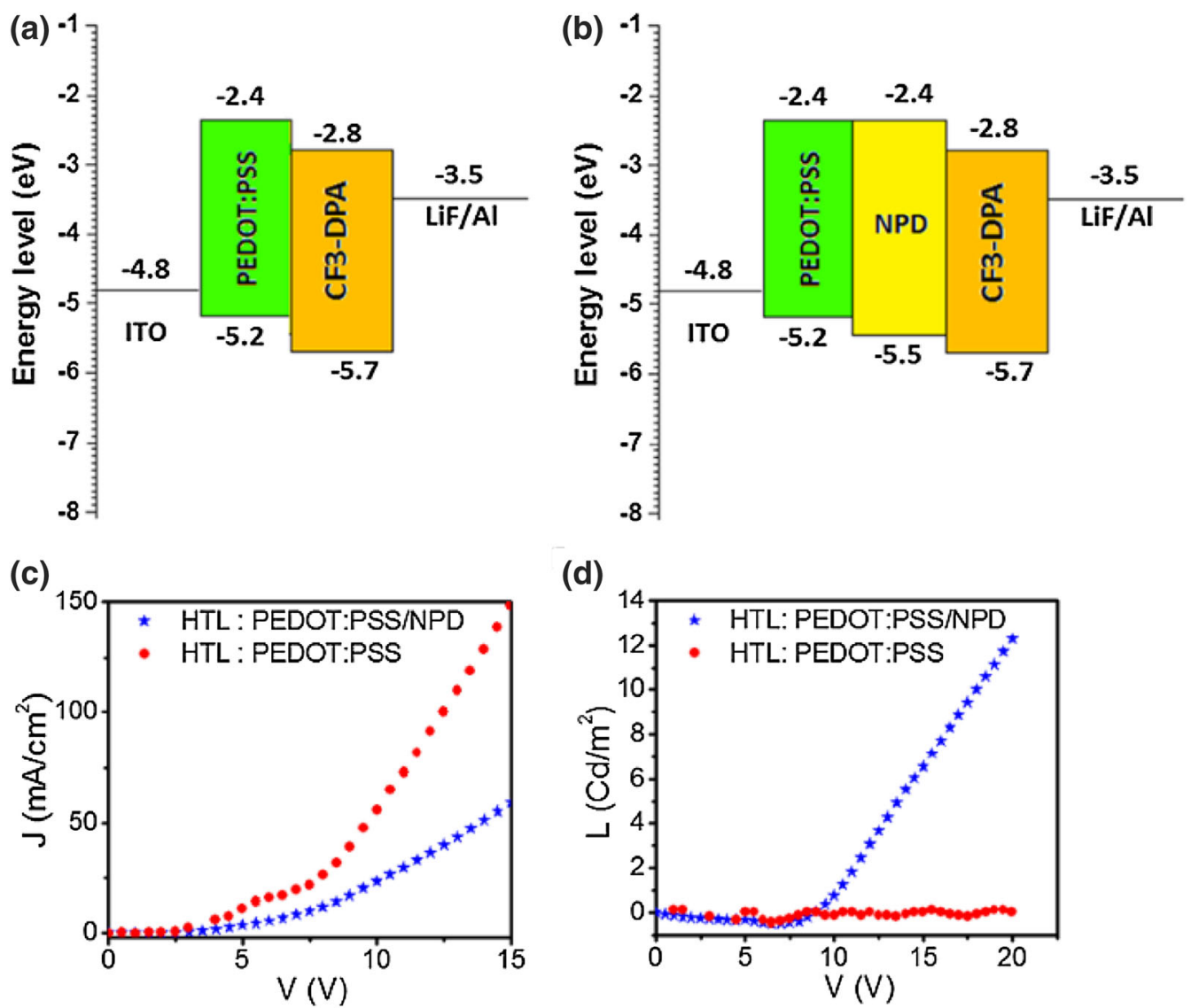

Figure 2. (a) Energy band diagram of ITO/PEDOT:PSS/CF3-DPA/LiF-Al device; (b) Energy band diagram of ITO/PEDOT:PSS/NPD/CF3-DPA/LiF-Al device; (c) Current density (J) vs Voltage (V) plot of ITO/PEDOT:PSS/CF3-DPA/LiF-Al (red circles) and ITO/PEDOT: PSS/NPD/CF3-DPA/LiF-Al (blue stars) devices; (d) Luminance (L) vs Voltage (V) plot of ITO/PEDOT:PSS/CF3-DPA/LiF-Al (red circles) and ITO/PEDOT:PSS/NPD/CF3-DPA/LiF-Al (blue stars). Devices showed higher light output in PEDOT:PSS/NPD based devices as compared to PEDOT:PSS based devices.

low $\mathrm{V}_{\mathrm{ON}}=4.0 \mathrm{~V}$ compared to $\mathrm{V}_{\mathrm{ON}}=10 \mathrm{~V}$ for the trilayer devices without PEDOT:PSS (Figure 3(a)). We observed that all CuPc-based devices had low current density. The light output for the multilayer device was $6 \mathrm{Cd} / \mathrm{m}^{2}$ at $10 \mathrm{~mA} / \mathrm{cm}^{2}$. The reason for the decrease in current density in $\mathrm{CuPc}$ based OLEDs has been discussed in the literature. ${ }^{27}$ It is believed that oxygen is released from ITO at a higher bias which diffuses into the CuPc layer. For higher CuPc thicknesses, the diffused oxygen results in a higher number of trap states for the holes. This reduces the hole mobility resulting in charge imbalance reducing light output as well as the current density.

As observed in all the devices studied so far, the presence of PEDOT:PSS reduces $\mathrm{V}_{\mathrm{ON}}$ as well as the light output. In addition, NPD/CF3-DPA interface was found to play a crucial role in light output. Therefore, we decided to fabricate bilayer devices with only NPD as the HTL and CF3-DPA as the emitting layer.
See schematic of the device in Figure 4(a) and the corresponding energy band diagram in Figure 4(b). The thickness of NPD was kept $\sim 45 \mathrm{~nm}$. As the $\mathrm{E}_{\text {Hомо }}$ and E $_{\text {LUMO }}$ of CF3-DPA were close to that of Alq3, devices with similar geometry but with Alq3 as the emitting layer were fabricated for comparison. The thickness of CF3-DPA was kept at $\sim 50 \mathrm{~nm}$ which was optimized to give the maximum light output in this geometry. The thickness of Alq3 was $\sim 60 \mathrm{~nm}$. Figure 4(c) shows the J-V plots of two separate devices with CF3-DPA and Alq3 as emitters. The $\mathrm{V}_{\mathrm{ON}}$ was observed to be higher in CF3-DPA than Alq3. Besides, the light output at $50 \mathrm{~mA} / \mathrm{cm}^{2}$ was almost one order of magnitude lower than that of Alq3 (Figure 4(d)). The electroluminescence spectra of Alq3 and CF3-DPA were recorded (Figure 4(e) and (f)) showing the emission peak at $514 \mathrm{~nm}$ and $439 \mathrm{~nm}$, respectively. Deep blue emission was observed from the CF3-DPA devices (see an image of the device in the inset of Figure 4(f)). The 1931 CIE 
(a)
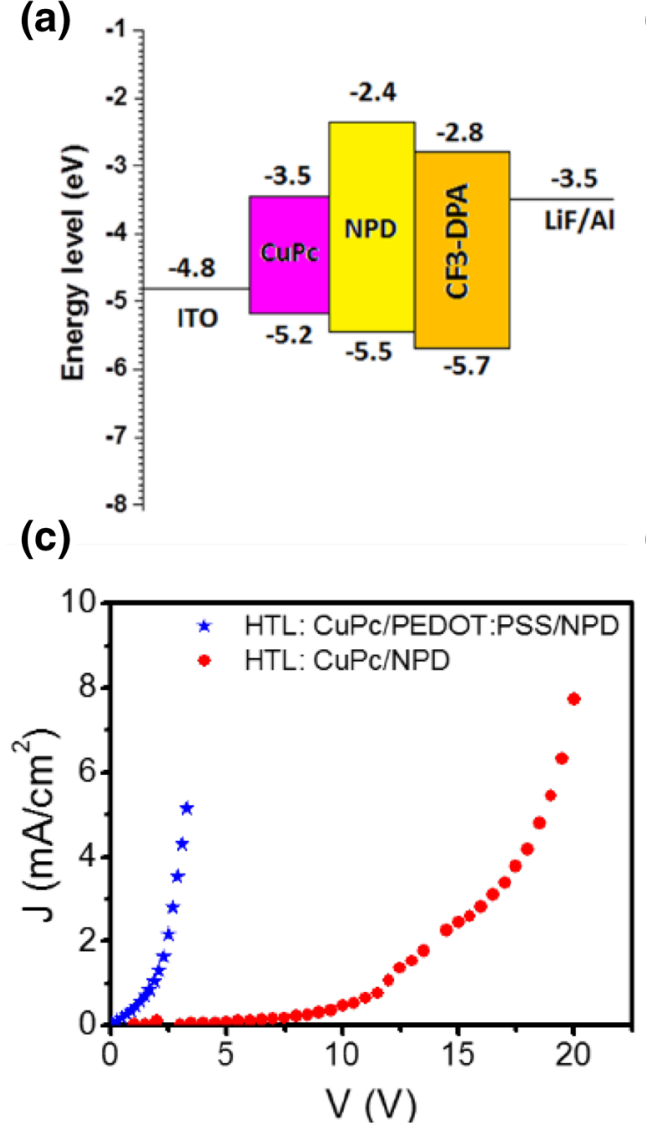

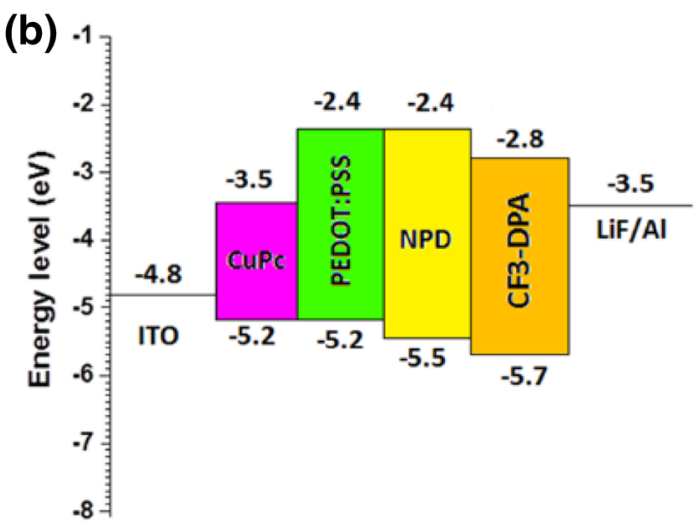

(d)

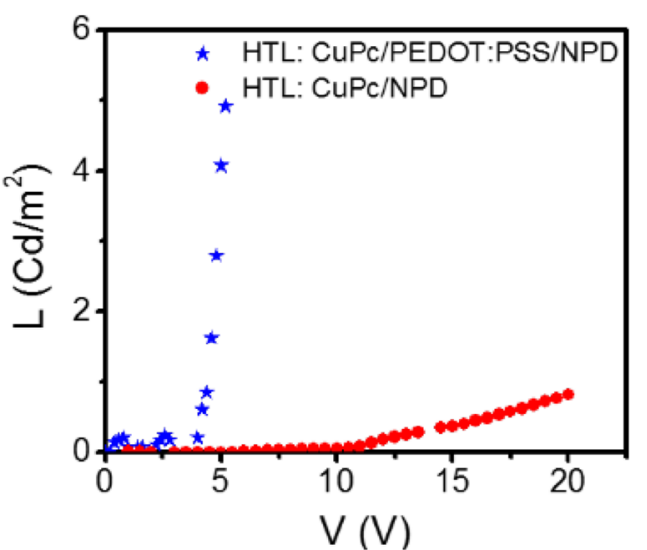

Figure 3. (a) Energy band diagram of ITO/CuPc/NPD/CF3-DPA/LiF-Al device; (b) Energy band diagram of ITO/CuPc/PEDOT:PSS/NPD/CF3-DPA/LiF-Al device; (c) J-V plot of ITO/ CuPc/NPD/CF3-DPA/LiF-Al (red circles) and ITO/CuPc/PEDOT:PSS/NPD/CF3-DPA/LiF-Al (blue stars). (d): L-V plot of ITO/CuPc/NPD/CF3-DPA/LiF-Al (red circles) and ITO/CuPc/ PEDOT:PSS/NPD/CF3-DPA/LiF-Al (blue stars).

chromaticity $(\mathrm{x}, \mathrm{y})$ was $(0.14,0.04)$. Considering that the spectral response of the human eye in blue is much lower than that of green (see standard photopic curve), the light output seems reasonable though not very promising. This could be attributed to primarily two factors: (i) Low internal quantum efficiency of CF3-DPA in the solid state; ${ }^{28}$ (ii) low probability of recombination in the emissive layer. In order to overcome the second problem, it was decided to introduce an ETL or a hole blocking layer (HBL) after CF3-DPA or introduce an electron blocking layer (EBL) before the CF3DPA. Bathophenanthroline (BPhen) with a HOMO of $-6.4 \mathrm{eV}$ and LUMO of $-3.0 \mathrm{eV}^{29}$ seemed to be a good choice for an HBL when placed after CF3-DPA. However, devices with ITO/NPD/CF3-DPA/BPhen/LiF-Al geometry did not help to improve the device performance. TPBi is also known to be an efficient HBL and ETL. ${ }^{30}$ Devices with the geometry ITO/NPD/CF3DPA/TPBi/LiF-Al were fabricated with the thickness of thermally evaporated TPBi being $\sim 15 \mathrm{~nm}$. The devices based on this geometry gave a high current density $(\mathrm{J})$ as well as high light output (L, Figure 5(b) and (c)) resulting in a luminance of $54 \mathrm{Cd} / \mathrm{m}^{2} @ 50 \mathrm{~mA} / \mathrm{cm}^{2}$. Since the LUMO of TPBi is just $0.1 \mathrm{eV}$ higher than that of CF3-DPA (Figure 5(a)), it efficiently transports the electrons to the emissive layer. In addition, it has a $\mathrm{HOMO}$ of $-6.2 \mathrm{eV}$ which helps to block the holes thereby increasing the efficiency of carrier recombination in the emissive layer. 4,4'-Bis ( $N$-carbazolyl)-1, $1^{\prime}$ biphenyl (CBP) is known to have ambipolar charge transport properties ${ }^{31}$ and has been mostly used as a host material for phosphorescent dopants. It has a HOMO of $-5.9 \mathrm{eV}$ and a LUMO of $-2.6 \mathrm{eV}$. Thus, we made devices where we used PEDOT:PSS and CBP as double HTLs with the following device geometry, ITO/PEDOT:PSS/CBP/CF3-DPA/TPBi/LiF-Al. In these devices, a $40 \mathrm{~nm}$ thick CBP layer was thermally evaporated over spin coated ITO/PEDOT:PSS. There was a weak blue emission from the devices with low currents. To possibly increase the efficiency of these devices, the area of contact of CBP with the active layer was increased. A blend of CF3-DPA and CBP (1:1 
(a)

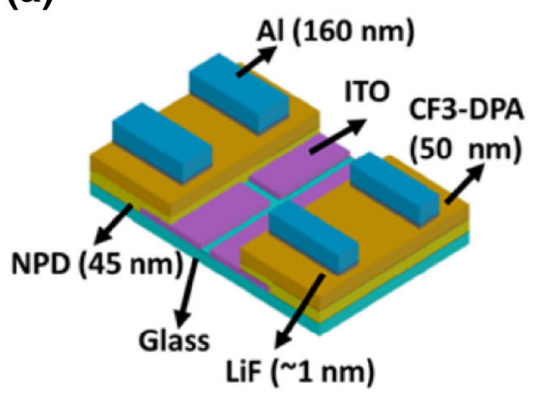

(b)

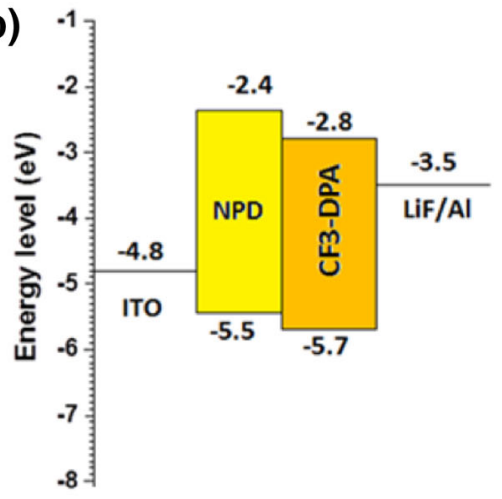

(c)

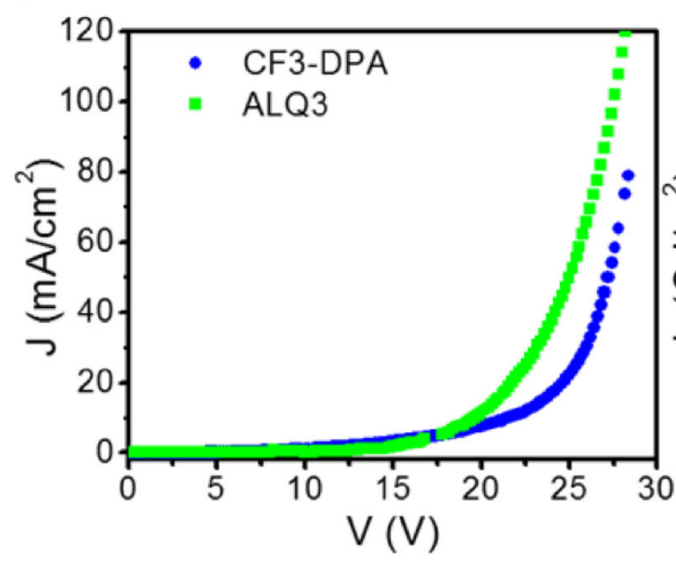

(d)

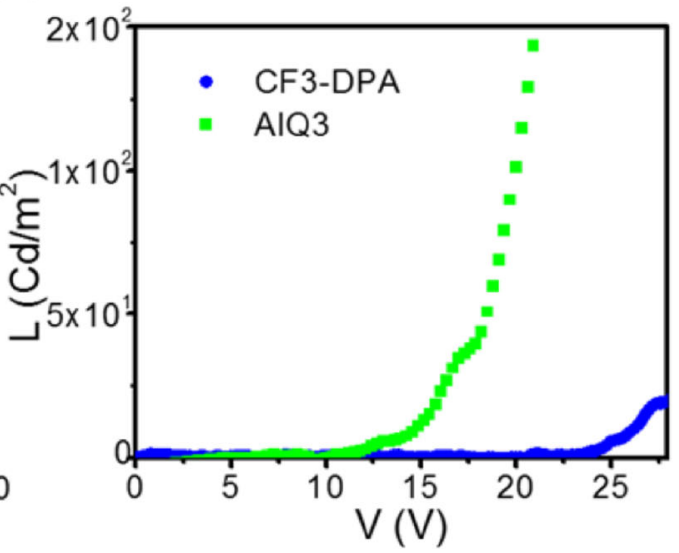

(d) (e)

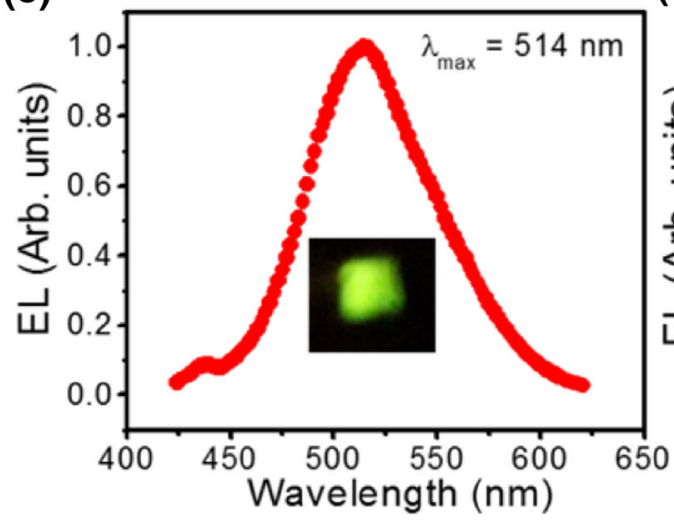

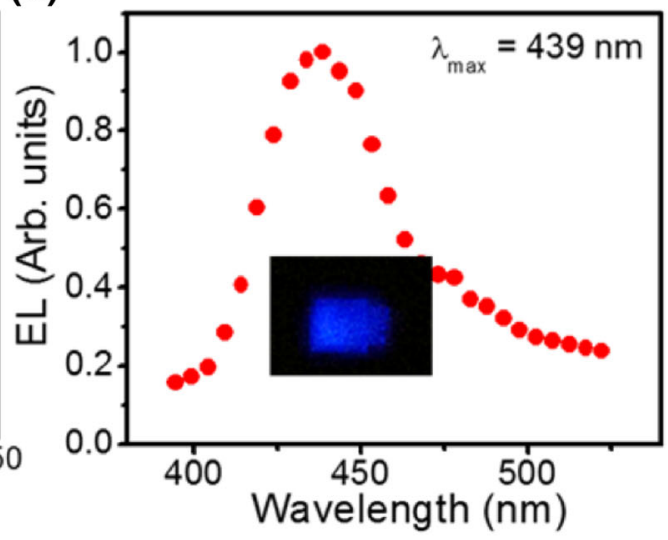

Figure 4. (a) 3D Schematic of ITO/NPD/CF3-DPA/LiF-Al OLED device; (b) Energy band diagram of the same device; (c) Current density (J) vs Voltage (V) plot of CF3-DPA (blue circles) and Alq3 (green squares); (d) Luminance (L) vs Voltage (V) plot of CF3-DPA (blue circles) and Alq3 (green squares); (e) Electroluminescence spectra of Alq3 showing emission peak at $515 \mathrm{~nm}$ (inset: image of the device emitting green light); (f) Electroluminescence spectra of CF3-DPA showing emission peak at $439 \mathrm{~nm}$ (inset: image of the device emitting blue light).

weight ratio) was made in chloroform and it was spin coated on ITO/PEDOT:PSS at $5000 \mathrm{rpm}$ for $40 \mathrm{~s}$ resulting in a thickness of $45 \mathrm{~nm}$. Devices with the geometry ITO/PEDOT:PSS/(CBP + CF3 - DPA)/TPBi/LiF-Al were fabricated which showed low $\mathrm{V}_{\mathrm{ON}}(\sim 5.5 \mathrm{~V})$ and a very sharp increase of light (Figure 5(b) and (c)).
However, these devices were highly unstable and the light emission sharply decreased at a $\mathrm{J}$ of $5 \mathrm{~mA} / \mathrm{cm}^{2}$. We tried to increase their stability, by changing the ratio of CBP and CF3-DPA (0.2:1 to $1: 1$ by weight), the thickness of the layer $(40-60 \mathrm{~nm})$ and doing postannealing of the CF3-DPA+CBP spin-coated layer at 


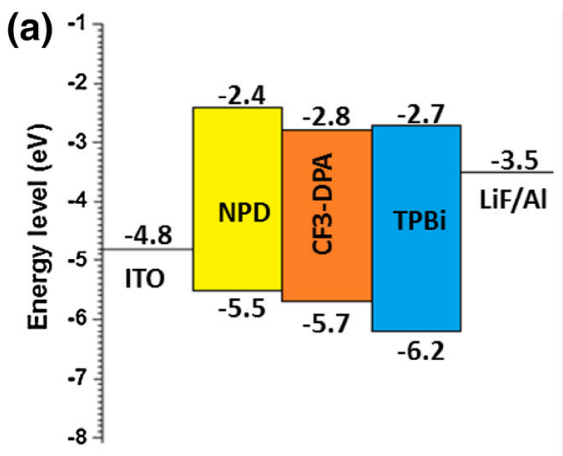

(b)

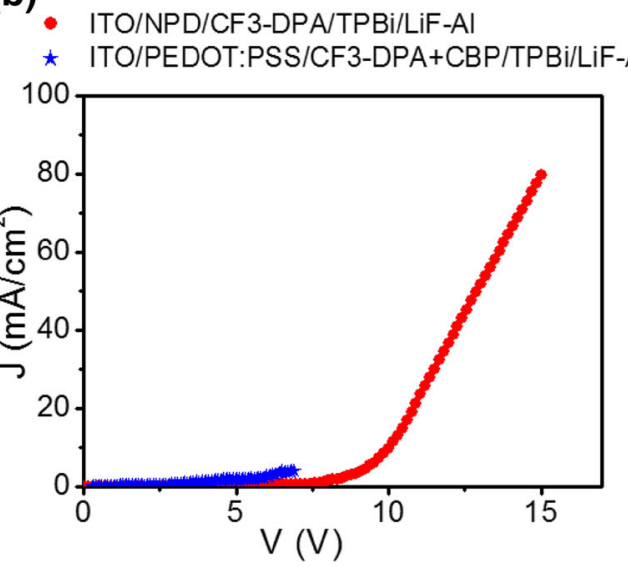

(c)

- ITO/NPD/CF3-DPA/TPBi/LiF-Al

- ITO/PEDOT:PSS/CF3-DPA+CBP/TPBi/LiF-AI

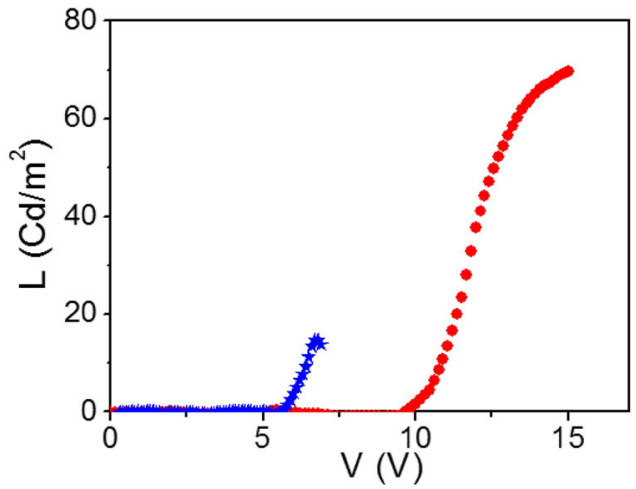

Figure 5. (a) Energy band diagram of ITO//NPD/CF3-DPA/TPBi/LiF-Al device; (b) J-V plot of ITO/NPD/CF3-DPA/TPBi/LiF-Al (red circles) and ITO/NPD/CF3-DPA+CBP/TPBi/LiF-Al (blue stars). (c) $\mathrm{L}-\mathrm{V}$ plot of ITO/NPD/CF3-DPA/TPBi/LiF-Al (red circles) and ITO/NPD/CF3-DPA+CBP/TPBi/LiF-Al (blue stars).

(a)

HTL: NPD

HTL: PEDOT:PSS/NPD

* HTL: CuPc/NPD

HTL: CUPc/PEDOT:PSS/NPD

- HTL:NPD, ETL:TPBI

HTL:PEDOT:PSS/CBP+CF3-DPA, ETL:TPBi

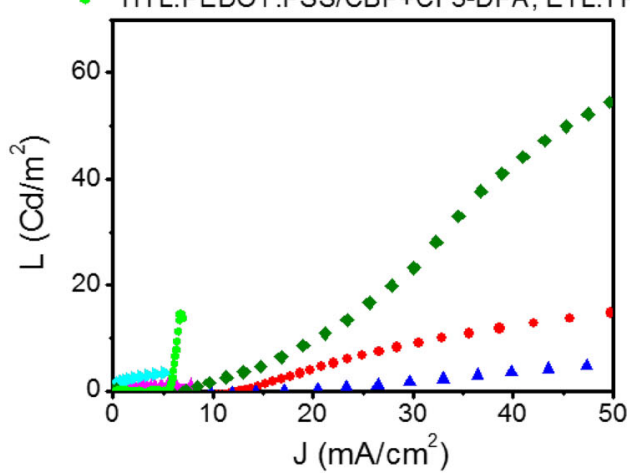

(b)
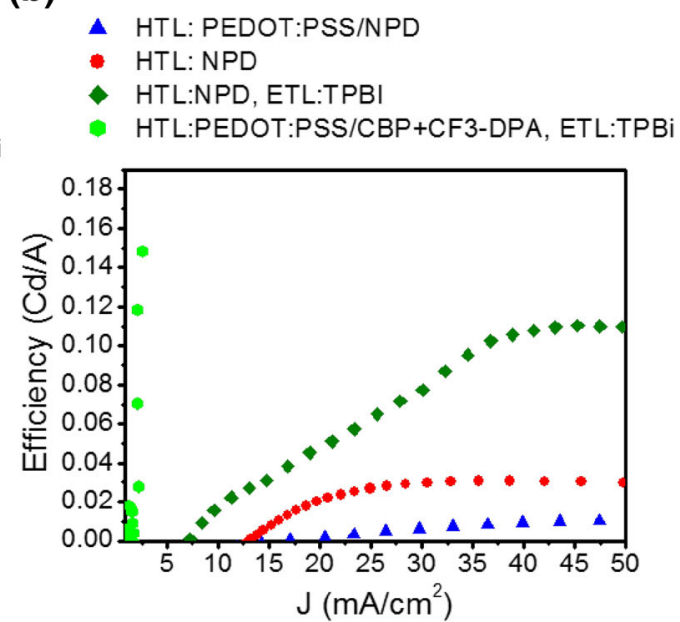

Figure 6. (a) L-J plot of the devices with all different geometries studied; (b) Efficiency (Cd/A) vs J plot for the four best devices. Red circles are for the bilayer device with NPD as HTL, blue triangles are for the tri-layer device with PEDOT:PSS/NPD as the HTL, dark green diamonds are for the trilayer device with NPD as HTL and TPBi as the ETL and the light green polygons are for the device where the active emissive layer is formed with a blend of CF3-DPA and CBP.

different temperatures $\left(100-125^{\circ} \mathrm{C}\right)$. The stability of the devices could not be improved appreciably. However, these results indicate that $\mathrm{CBP}$ behaves as an efficient carrier transporter/blocker.
To compare devices with all geometries, we plotted $\mathrm{L}$ vs $\mathrm{J}$ for all of them which is shown in Figure 6(a). It is clear from the plot that the best device geometry is ITO/NPD/CF3-DPA/TPBi/LiF-Al as it gives 
the highest efficiency at $50 \mathrm{~mA} / \mathrm{cm}^{2}$ (Figure 6(b)). However, it is worth noting that at low $\mathrm{J}\left(\sim 1 \mathrm{~mA} / \mathrm{cm}^{2}\right)$, the devices ITO/PEDOT:PSS/(CBP+CF3-DPA)/TPBi/ LiF-Al seemed to be the most efficient thereby showing the utility of CBP as an ambipolar charge transporter.

\section{Conclusions}

We have synthesized trifluoromethyl substituted 1,8diaryl anthracene (CF3-DPA) which absorbs at $394 \mathrm{~nm}$ and emits at $432 \mathrm{~nm}$ in solution. It shows a quantum efficiency of $67 \%$ when compared to 9,10-DPA thereby making it a promising candidate for blueemitting OLEDs. Furthermore, in solid state thin films, it shows a small red shift in emission. The morphology of the films shows a low surface roughness indicating the possibility of the formation of smooth interfaces with HTLs in OLED devices. OLED devices with different geometries containing different HTLs were fabricated by vacuum thermal deposition. The EL spectra obtained on the working devices showed a peak at $439 \mathrm{~nm}$. The turn on voltages and luminance values were compared for the devices with different geometries. Bilayer devices with PEDOT:PSS as the HTL gave no light output. Including a layer of NPD in the devices made them emit light indicating that the NPD/CF3-DPA interface was important for light output. In addition, the turn on voltage was also low $\sim 7.5 \mathrm{~V}$ with PEDOT:PSS. The performance of $\mathrm{CuPc}$ based devices was poor. However, bilayer devices with the following geometry of ITO/NPD/CF3-DPA/LiF-Al gave reasonable light output though the turn on voltage was slightly higher at $13 \mathrm{~V}$. Furthermore, introducing charge carrier blockers/transporters like TPBi and CBP helped to increase the efficiency of the devices. Devices having ITO/NPD/CF3-DPA/TPBi/LiF-Al geometry were observed to be the most efficient with a $\mathrm{V}_{\mathrm{ON}} \sim 8.5 \mathrm{~V}$. From our studies, we conclude that though a derivative of 1,8-DPA can be used as a blue emitter in OLED devices, the performance of the devices is limited by the mismatch of the hole and electron mobilities and the low internal quantum efficiency of CF3-DPA in solid state. Optimized device geometry using novel, suitable carrier blockers might increase the efficiency of 1,8-di-(4trifluromethylphenyl)-anthracene based blue emitters.

\section{Acknowledgements}

We thank Swati Dixit and Pradnya Parab for their help in the synthesis of the organic molecule and fabrication of the devices. We would also like to thank Prof. Sandip Ghosh who provided us access to the electroluminescent setup. Vivas Bagwe and John Jesudesan are acknowledged for AFM and film thickness measurements.

\section{References}

1. (a) Forrest S R 2004 The path to ubiquitous and low-cost organicelectronic appliances on plastic Nature 428 911;(b) Reineke S, Thomschke M, Luessem B and Leo K 2013 White organic light-emitting diodes: Status and perspective Rev. Mod. Phys. 851245

2. Geffroy B, Roy P le and Prat C 2006 Organic lightemitting diode (OLED) technology: Materials, devices and display technologies Polym. Int. 55572

3. Bruetting W, Frischeisen J, Schmidt T D, Scholz B J and Mayr C 2013 Device efficiency of organic light-emitting diodes: Progress by improved light outcoupling Phys. Status Solidi A 21044

4. (a) Xu R-P, Li Y-Q and Tang J-X 2016 Recent advances in flexible organic light-emitting diodes J. Mater. Chem. C 4 9116; (b) Nayak P K, Agarwal N, Ali F, Patankar M, Periasamy N and Narasimhan K L 2010 Blue and white light electroluminescence in a multilayer OLED using a new aluminium complex J. Chem. Sci. 122847

5. (a) Kuma H and Hosokawa C 2014 Blue fluorescent OLED materials and their application for highperformance devices Sci. Technol. Adv. Mater. 15 034201; (b) Bala I, Ming L, Yadav R A K, De J, Dubey D K, Kumar S, Singh H, Jou J-H, Kailasam K and Pal S K 2018 Deep-blue OLED fabrication from heptazine columnar liquid crystal based AIE-active sky-blue emitter Chemistry Select 37771

6. Shin H, Lee J-H, Moon C-K, Huh J-S, Sim B and Kim J-J 2016 sky-blue phosphorescent OLEDs with $34.1 \%$ external quantum efficiency using a low refractive index electron transporting layer $A d v$. Mater. 28 4920

7. (a) Agarwal N, Nayak P K and Periasamy N 2008 Synthesis, photoluminescence and electrochemical properties of 2,7-diarylfluorene derivatives J. Chem. Sci. 120 355; (b) Nayak P K, Agarwal N and Periasamy N 2010 Synthesis, photophysical and electrochemical properties of 2,8-diaryl-dibenzothiophene derivatives for organic electronics J. Chem. Sci. 122119

8. Adachi C, Tsutsui T and Saito S 1990 Blue light-emitting organic electroluminescence devices Appl. Phys. Lett. 56799

9. Becker H-D 1993 Unimolecular photochemistry of anthracenes Chem. Rev. 93145

10. Serevičius T, Komskis R, Adomènas P, Adomėniene O, Jankauskas V, Gruodis A, Kazlauskas K and Juršènas S 2014 Non-symmetric 9,10-diphenylanthracene-based deep-blue emitters with enhanced charge transport properties Phys. Chem. Chem. Phys. 167089

11. Wu C-L, Chang C-H, Chang Y-T, Chen C-T, Chen C-T and $\mathrm{Su}$ C-J 2014 High efficiency non-dopant blue organic light emitting diodes based on anthracene-based fluorophores with molecular design of charge transport and red-shifted emission proof J. Mater. Chem. C 27188

12. Ni S Y, Wang X R, Wu Y Z, Chen H Y, Zhu W Q, Jiang X Y, Zhang Z L and Sun R G 2004 Decay mechanisms of a blue organic light emitting diode Appl. Phys. Lett. 85878

13. Shi J and Tang C W 2002 Anthracene derivatives for stable blue-emitting organic electroluminescence devices Appl. Phys. Lett. 803201 
14. Kim S-K, Yang B, Ma Y, Lee J-H and Park J-W 2008 Exceedingly efficient deep-blue electroluminescence from new anthracenes obtained using rational molecular design J. Mater. Chem. 183376

15. Morris J V, Mahaney M A and Huber J R 1976 Fluorescence Quantum Yield Determinations. 9,10Diphenylanthracene as a Reference Standard in Different Solvents J. Phys. Chem. 80969

16. Klauk H, Zschieschang U, Weitz R T, Meng H, Sun F, Nunes G, Keys D E, Fincher C R and Xiang Z 2007 Organic Transistors Based on Di-(phenylvinyl)anthracene: Performance and stability Adv. Mater. 19 3882

17. Meng H, Sun F, Goldfinger M B, Jaycox G D, Li Z, Marshall W J and Blackman G S 2005 High-performance, stable organic thin-film field-effect transistors based on Bis-5-alkylthiophen-2c-yl-2,6-anthracene semiconductors J. Am. Chem. Soc. 1272406

18. Jiang L, Hu W, Wei Z, Xu W and Meng H 2009 High performance organic single-crystal transistors and digital inverters of an anthracene derivative $A d v$. Mater. 21 3649

19. Liu J, Dong H, Wang Z, Ji D, Cheng C, Geng H, Zhang H, Zhen Y, Jiang L, Fu H, Bo Z, Chen W, Shuai Z and Hu W 2015 Thin film field-effect transistors of 2,6-diphenyl anthracene (DPA) Chem. Commun. 5111777

20. Lynch P J, O’Neill L, Bradley D, Byrne H J and McNamara M 2007 Systematic study of the effects of naphthalene and anthracene substitution on the properties of PPV derivative conjugated systems Macromolecules 40 7895

21. Agarwal N, Patil M and Patil M 2015 Synthesis of highly emissive 1,8-diaryl anthracene derivatives and fabrication of their micro/nanostructures RSC Adv. 598447

22. So S K, Choi W K, Cheng C H, Leung L M and Kwong C F 1999 Surface preparation and characterization of indium tin oxide substrates for organic electroluminescent devices Appl. Phys. A 68447

23. $\mathrm{Mu} \mathrm{H}$, Li W, Jones R, Steckl A and Klotzkin D 2007 A comparative study of electrode effects on the electrical and luminescent characteristics of Alq3/TPD OLED: Improvements due to conductive polymer (PEDOT) anode J. Lumin. 126225

24. Hung L S and Chen C H 2002 Recent progress of molecular organic electroluminescent materials and devices Mater. Sci. Eng. R 39143

25. Groenendaal L B, Jonas F, Freitag D, Pielartzik H and Reynolds J R 2000 Poly-(3,4 ethylenedioxythiophene) and its Derivatives: Past, present, and future Adv. Mater. 12481

26. Tutis E, Berner D and Zuppiroli L 2003 Internal electric field and charge distribution in multilayer organic light emitting diodes J. Appl. Phys. 934594

27. Tadayyon S M, Grandin H M, Griffiths K, Norton P R, Aziz H and Popovic Z D 2004 CuPc buffer layer role in OLED performance: A study of the interfacial band energies Org. Electron. 5157

28. Manna B, Ghosh R and Palit D K 2015 exciton dynamics in anthracene nanoaggregates J. Phys. Chem. C 119 10641

29. Yoon J A, Kim Y H, Kim N H, Yoo S, Lee S Y, Zhu F R and Kim W Y 2014 Highly efficient blue organic light-emitting diodes using quantum well-like multiple emissive layer structure Nanoscale Res. Lett. 9 191

30. Kulkarni A P, Tonzola C J, Babel A and Jenekhe S A 2004 Electron transport materials for organic light-emitting diodes Chem. Mater. 164556

31. Adachi C, Kwong R and Forrest S R 2001 Efficient electrophosphorescence using a doped ambipolar conductive molecular organic thin film Org. Electron. 2 37 